\title{
REVIEWERS
}

\section{Guest Reviewers, Journal of Classification Volume 34, 2017}

In addition to the members of the Editorial Board, the individuals listed below refereed manuscripts that were submitted to the journal. Their assistance is gratefully acknowledged.

$\begin{array}{ll}\text { Ahmed N. Albatineh } & \text { Hans-Friedrich Köhn } \\ \text { Andres Alonso } & \text { Robert Krafty } \\ \text { K.T. Atanassov } & \text { Herbert Lee } \\ \text { Apostolos Batsidis } & \text { Pierre Legendre } \\ \text { Daniel Bauer } & \text { Rosaria L. Lombardo } \\ \text { Patrice Bertrand } & \text { Elizabeth A. Maharaj } \\ \text { Anne-Laure Boulesteix } & \text { Vladimir Makarenkov } \\ \text { Laine Bradshaw } & \text { Dan McNeish } \\ \text { Michael Brusco } & \text { Paul McNicholas } \\ \text { Pedro Contreras } & \text { Mhamed Mesfioui } \\ \text { Renato Cordeiro de Amorim } & \text { Seyedali Mirjalili } \\ \text { Steven Culpepper } & \text { Boris Mirkin } \\ \text { Bijan Davvaz } & \text { Fionn Murtagh } \\ \text { John Daws } & \text { Janmenjoy Nayak } \\ \text { Jimmy de la Torre } & \text { Elena Rubei } \\ \text { George Diekhoff } & \text { Stanley L. Sclove } \\ \text { Mohamed Elhoseny } & \text { Peter Scully } \\ \text { Kostantinos Fokianos } & \text { Emilie Shireman } \\ \text { Stephen France } & \text { Jordan Stevens } \\ \text { Andrew Francis } & \text { Clintin Stober } \\ \text { Kiero Guerra } & \text { Zdenek Sulc } \\ \text { Michel Habib } & \text { Thaddeus Tarpey } \\ \text { Jeffrey Harring } & \text { Marieke E. Timmerman } \\ \text { Christian Hennig } & \text { Valentin Todorov } \\ \text { Michaela Hoffman } & \text { Jeroen Vermunt } \\ \text { Wen-Liang Hung } & \text { Sunny Wang } \\ \text { Huengsun Hwang } & \text { Matthijs J. Warrens } \\ \text { Harry Joe } & \text { Naoto Yamashita } \\ \text { Yurij Kharin } & \text { Alexandr Zubarev } \\ & \end{array}$

